\title{
Sensor Based Rehabilitation Tool for Epilepsy Patients
}

\author{
T.Manoj Prasath, Prasath Alias Surendhar, R.Kishore Kanna, Vasukidevi Ramachandran
}

\begin{abstract}
Epilepsy is a neurological issue set apart by abrupt intermittent scenes of tactile unsettling influence, loss of cognizance, or seizures, related with unusual electrical movement in the mind. Seizures can impact any procedures in the directions of our mind, there is a transient perplexity, memory misfortune and lost awareness. Epilepsy has no recognizable reason in about portion of those with the condition, the condition might be followed to different variables head injury, mind wounds, hereditary impact. Such injuries have ended up being not kidding and lethal much of the time, to maintain a strategic distance from such full of feeling mishaps, the reason for this undertaking is to propose a (model) gadget which will estimate the physiological changes in the human body before the repetitive scenes of seizures will be checked. The patient will be advised preceding the loss of cognizance and tangible unsettling influence. The gadget will detect the limit estimation of the customized physiological parameter, on the increase of this edge a caution or a notice will be gotten from the gadget, this will guarantee a conceivable recovery to a patient. Preventive measures to a patient can be taken to verify any unexpected incidental injury.
\end{abstract}

\section{INTRODUCTION}

Epilepsy is a standout amongst the most common neurological issue in human beings.it is described seizures in which typical electrical action in the cerebrum is translated and causes the loss of awareness or an entire body spasm. Patients are ignorant of the event of seizure because of the irregular idea of them which may expand the danger of physical damage or may prompt uneven accidents.Studies demonstrate that $4-5 \%$ of the absolute total populace has been experiencing epilepsy. Electroencephalogram is one of the significant instruments for conclusion and investigation of epilepsy. Electroencephalogram is the recorded portrayal of electrical action delivered by terminating of neuron inside the cerebrum along the scalp. For chronicle of EEG, cathodes will be stuck at some key focuses on the patient's head. Cathodes get the sign and will be recorded in a chronicle gadget through wires which are associated with terminals, As complete visual investigation of EEG sign is troublesome, programmed location is liked. Fourier change has been most normally utilized in beginning of preparing of EEG signals. The mechanized analysis of epilepsy can be subdivided into pre-preparing, highlight extraction, and order. Seizure identification can be delegated either seizure

Revised Manuscript Received on July 18, 2019.

T.Manoj Prasath, Dept Of Biomedical Engg, BIHER, Chennai, Tamilnadu,India.

Prasath Alias Surendhar, Dept Of Biomedical Engg, BIHER, Chennai, Tamilnadu, India.

R.Kishore Kanna, Dept Of Biomedical Engg, BIHER, Chennai, Tamilnadu, India.

Vasukidevi Ramachandran, Dept Of Biomedical Engg, BIHER, Chennai, Tamilnadu, India.

\section{Keywords: Seizures; Sensor; Thresholdvalue; Rehabilitation}

beginning recognition or seizure occasion discovery. In seizure beginning discovery the intention is to perceive the beginning of seizure with the most brief conceivable deferral. The motivation behind seizure occasion identification is to distinguish seizures with the most elevated conceivable exactness.

\section{METHODOLOGY \& TEST RESULTS}

The gadget can estimate physiological changes before the epilepsy seizures, The model comprises of a sensor, the sensor will recognize any unusual changes in the physiological parameters in the patient preceding the seizure reoccurrence, when the customized limit will be crossed this will buzz an alert in the gadget and the wellbeing estimates will be taken to the patient.

There are mostly two types of components that will be used in this project.

1. Sensors

2. Buzzers

\section{Sensor:}

A sensor is an electronic item whose object is to distinguish the occasions or change in its condition and after that give a relating yield.

Sensor is a sort of transducer which produces different kinds of yield yet the most sorts of yields it produce is electrical and optical (light based).

\section{Use Of Sensor:}

A sensor can be utilized to recognize the change, the typical fundamental action in the body, it alludes to the identification of the anomalous conduct of the physiological parameters of a human body of the patient, the model depends on the implanted programming which depends on the set edge, a predisposition will be set for the sensor.

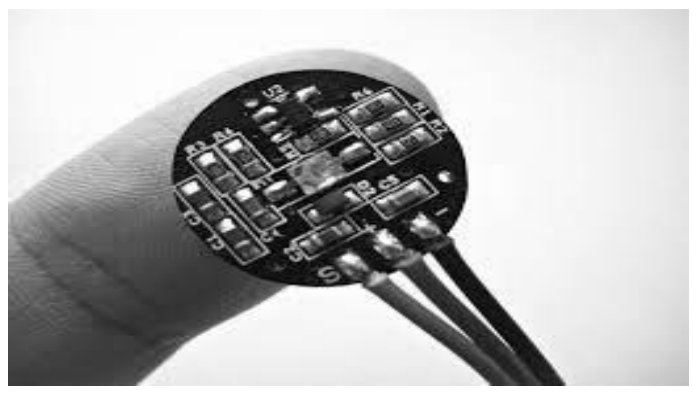

Fig:1 Pulse sensor.

Published By: 


\section{Buzzer:}

A buzzer is an electronic device that gives a buzzing noise, as an alarm which is used for signalling purpose.

\section{FUTURE WORK}

- $\quad$ Looking for preferable sensor to detect the change in vital parameter.

- $\quad$ Programming the sensor using embedded programming.

$\bullet$

Testing the device to forecast the seizure

attack.

\section{REFERENCES}

1. O'donoghue MF, Goodridge DM, Redhead K, Sander JW, Duncan JS. Assessing the psychosocial consequences of epilepsy: a community-based study. Br J Gen Pract. 1999 Mar 1;49(440):211-4.

2. Fairgrieve SD, Jackson M, Jonas P, Walshaw D, White K, Montgomery TL, Burn J, Lynch SA. Population based, prospective study of the care of women with epilepsy in pregnancy. Bmj. 2000 Sep 16;321(7262):6745.

3. Nicoletti A, Sofia V, Biondi R, Fermo SL, Reggio E, Patti F, Reggio A. Epilepsy and multiple sclerosis in Sicily: a population-based study. Epilepsia. 2003 Nov;44(11):1445-8.

4. Winkler AS, Kerschbaumsteiner K, Stelzhammer B, Meindl M, Kaaya J, Schmutzhard E. Prevalence, incidence, and clinical characteristics of epilepsy-A community-based door-to-door study in northern Tanzania. Epilepsia. 2009 Oct;50(10):2310-3.

5. Chang YT, Chen PC, Tsai IJ, Sung FC, Chin ZN, Kuo HT, Tsai CH, Chou IC. Bidirectional relation between schizophrenia and epilepsy: a population-based retrospective cohort study. Epilepsia. 2011 Nov;52(11):2036-42.

6. Oka E, Ohtsuka Y, Yoshinaga H, Murakami N, Kobayashi K, Ogino T. Prevalence of childhood epilepsy and distribution of epileptic syndromes: a population-based survey in Okayama, Japan. Epilepsia. 2006 Mar;47(3):626-30.

7. Qin $\mathrm{P}, \mathrm{Xu} \mathrm{H}$, Laursen TM, Vestergaard M, Mortensen PB. Risk for schizophrenia and schizophrenia-like psychosis among patients with epilepsy: population based cohort study. Bmj. 2005 Jun 30;331(7507):23.

8. Berg AT, Langfitt JT, Testa FM, Levy SR, DiMario F, Westerveld M, Kulas J. Global cognitive function in children with epilepsy: a community-based study. Epilepsia. 2008 Apr;49(4):608-14.

9. Koponen A, Seppälä U, Eriksson K, Nieminen P, Uutela A, Sillanpää M, Hyvärinen L, Kälviäinen R. Social Functioning and Psychological Well-Being of 347 Young Adults with Epilepsy Only-Population-Based, Controlled Study from Finland. Epilepsia. 2007 May;48(5):907-12.

1. Lim LL, Foldvary N, Mascha E, Lee J. Acetazolamide in women with catamenial epilepsy. Epilepsia. 2001 Jun;42(6):746-9 